«Ärztliche Zeugnisse, Berichte und Gutachten sind Urkunden. Bei deren Ausstellung haben Arzt und Ärztin alle Sorgfalt anzuwenden, um nach bestem Wissen ihre ärztliche Überzeugung auszudrücken.» So steht es in der FMH-Standesordnung [1].

Klar ist, dass der Arzt in Bericht, Zeugnis oder Gutachten die Gesundheitssituation für den Patienten oder Exploranden fair darstellen muss. Er würde sich sonst dem Patienten gegenüber haftpflichtig machen.

Klar ist aber auch, dass Zeugnisse, Berichte und Gutachten, die die Situation für den Patienten ungerechtfertigt vorteilhaft erscheinen lassen, als falsches ärztliches Zeugnis strafbar sind. Wes- halb? Objektive ärztliche Zeugnisse und Berichte sind für das Funktionieren unserer Gesellschaft so wichtig, dass der Gesetzgeber sie strafrechtlich abgesichert hat.

Der Beitrag von Cornelia Dürr Brunner, Rechtsanwältin bei der AXA Winterthur, berichtet über zwei kürzliche Verurteilungen durch das Bundesgericht. Er kann Arzt und Ärztin möglicherweise helfen, die geltenden Spielregeln auch Patienten zu erklären, die meinen, ihr Arzt dürfe und solle ihnen mit einem Zeugnis «helfen», und nicht wahrhaben wollen, dass er objektiv berichten muss.

Hanspeter Kuhn, stv. Generalsekretär FMH

\title{
Strafrechtliche Relevanz von medizinischen Berichten
}

\begin{abstract}
Ärzte haben beim Verfassen ihrer medizinischen Berichte eine grosse Verantwortung, weil der Inhalt eines Gutachtens weitreichende Konsequenzen haben kann und sich spätere Akteure auf die medizinischen Angaben sollen verlassen können. Unterstrichen wird dies durch zwei neuere höchstrichterliche Entscheide, in denen Ärzte strafrechtlich verurteilt wurden, weil sie im Zusammenhang mit Leistungsansprüchen ihrer Patienten unwahre medizinische Aussagen gemacht hatten.*
\end{abstract}

Cornelia Dürr Brunner

\footnotetext{
* Diese Verurteilungen erfolgten aufgrund von Art. 318 Ziff. 1 Abs. 1 StGB (Urteil 6B_99/2008 vom 18. März 2008 sowie Urteil 6B_152/2007 vom 13. Mai 2008.
}

Korrespondenz:

Cornelia Dürr Brunner, lic. iur. AXA Winterthur General-Guisan-Strasse 40 CH-8401 Winterthur cornelia.duerr@gmx.ch
Jeder Arzt kommt im Verlaufe seiner Tätigkeit in die Lage, für seinen Patienten ärztliche Zeugnisse, Arztberichte oder Gutachten auszustellen. Dabei ist allgemein bekannt, dass diese Beurteilungen des Arztes für den Patienten - aber auch für Dritte - weitreichende Konsequenzen haben können. Namentlich in Haftpflicht- oder Sozialversicherungsverfahren spielen medizinische Aussagen eine entscheidende Rolle. So sind gerade für die juristische Beurteilung von Arbeitsunfähigkeit und Kausalität die vom Arzt gemachten Angaben oftmals unerlässlich und beweisrechtlich von grosser Bedeutung. Dies gilt nicht nur für die eigentlichen Gerichtsgutachten, in denen das Gericht den ärztlichen Gutachter offiziell auswählt und sich aufgrund eines speziellen Fragenkatalogs ein Bild machen möchte. Vielmehr kann bereits ein einfaches ärztliches Zeugnis von rechtlichem Belang für allfällige Leistungsansprüche des Patienten sein.

Weitgehend bekannt ist auch, dass im Zusammenhang mit der Erstellung von ärztlichen Begutachtungen straf- und datenschutzrechtliche Bestimmungen $\mathrm{zu}$ beachten sind. Im Vordergrund steht dabei vor allem die Berufsgeheimnisbestimmung von Art. 321 StGB, wonach in erster Linie das Arzt-Patienten-Verhältnis geschützt werden soll. Weniger bekannt ist hingegen, dass auch die inhaltliche Richtigkeit eines Zeugnisses, Berichtes oder Gutachtens strafrechtli-

\section{Importance pénale des rapports médicaux}

Les médecins ont une grande responsabilité lorsqu'ils rédigent des rapports médicaux, car le contenu d'une expertise peut avoir d'importantes conséquences et il faut ensuite pouvoir se fier aux indications médicales qui y figurent. C'est ce que souligne le Tribunal fédéral par deux arrêts récents où des médecins ont été condamnés pour avoir fourni des indications médicales contraires à la vérité en lien avec la prise en charge de prestations fournies à leurs patients. Ces condamnations se fondent sur l'article 318, al. 1 du Code pénal suisse (arrêt 6B_99/2008 du 18 mars 2008 et arrêt 6B_152/2007 du13 mai 2008).

chen Bestimmungen unterworfen ist. So sieht Art. 318 des Schweizerischen Strafgesetzbuches folgendes vor: «Ärzte, Zahnärzte, Tierärzte und Hebammen, die vorsätzlich ein unwahres Zeugnis ausstellen, das zum Gebrauche bei einer Behörde oder zur Erlangung eines 
unberechtigten Vorteils bestimmt, oder das geeignet ist, wichtige und berechtigte Interessen Dritter zu verletzen, werden mit Freiheitsstrafe bis zu drei Jahren oder Geldstrafe bestraft. Hat der Täter dafür eine besondere Belohnung gefordert, angenommen oder sich versprechen lassen, so wird er mit Freiheitsstrafe bis zu drei Jahren oder Geldstrafe bestraft. Handelt der Täter fahrlässig, so ist die Strafe Busse.» $\mathrm{Zu}$ diesem Straftatbestand gab es bislang selten Gerichtsurteile und wenig Literatur. Nun wurden vom Bundesgericht aufgrund dieser Bestimmung zwei Ärzte strafrechtlich verurteilt.

\section{Urteil 6B 99/2008 vom 18. März 2008}

Zuhanden der Eidgenössischen Invalidenversicherung hatte ein Arzt in den Jahren 1991 und 1995 für seine Patientin im Rahmen eines Rentenverfahrens ärztliche Berichte verfasst. Darin wies der Arzt auf die deutlich depressive Veranlagung und die ungünstigen familiären Verhältnisse (Alkoholabhängigkeit des Ehemannes) der Patientin hin. Ebenfalls sprach er von «weichteilrheumatischen Beschwerden und Magenbeschwerden, welche als Ausdruck der endogenen Depression somatisiert zu betrachten sind».

1996 erlitt die Patientin einen Autounfall. Gegenüber der Haftpflichtversicherung schrieb der Arzt im Jahr 2001 in einem Gutachten, dass seine Patientin (vor dem massgebenden Unfall 1996) nie Anzeichen einer depressiven Erkrankung gehabt und sich «psychopathologisch in einem völlig ausgeglichenen $\mathrm{Zu}$ stand» befunden hätte. Die Vorinstanz war nach Würdigung zahlreicher (anderer) ärztlicher Zeugnisse davon ausgegangen, dass die Berichte von 1991 und 1995 wahr waren, und ein klarer Zusammenhang zwischen der Arbeitsunfähigkeit der Patientin und der Depression bestanden hatte. Dies entspreche auch der Überzeugung des Arztes, der unglaubwürdig sei, wenn er behaupte, das IV-Rentenverfahren sei ihm nicht mehr bewusst gewesen. So habe es sich um eine langjährige Patientin des Arztes gehandelt, die regelmässig bei ihm in Konsultation gewesen sei. Das Bundesgericht räumte zwar ein, die Frage nach der Wahrheit eines Gesundheitszeugnisses habe gezwungenermassen eine subjektive Komponente, weil der Arzt einen Sachverhalt interpretieren müsse. Im vorliegenden Fall sei aber der Arzt nicht der Ansicht gewesen sei, seine Angaben von 2001 würden zutreffen.

Ebenfalls sei der Arzt als Spezialarzt für Psychiatrie und Psychotherapie mit der Begutachtung beauftragt worden, um Versicherungsansprüche aus dem Unfallereignis zu prüfen. Weil das ärztliche Zeugnis fälschlicherweise fast sämtliche Gesundheitsschädigungen dem Unfall und keine dem Vorzustand zuschreibe, sei es dazu bestimmt, unberechtigte Vorteile zu erlangen. Als langjährigem Gutachter habe dies dem Arzt bewusst gewesen sein müssen. Der Arzt wurde zu einer bedingt vollziehbaren Geldstrafe von 25 Tagessätzen zu Fr. 900.- verurteilt.

\section{Urteil 6B 152/2007 vom 13. Mai 2008}

Ein Psychiater hatte gegenüber der Krankentaggeldversicherung seiner Patientin eine vollständige Arbeitsunfähigkeit aufgrund einer Depression, von Schlaflosigkeit, hormonellen Störungen sowie Fettsucht attestiert. In der Zwischenzeit hatte seine Patientin eine IV-Rente beantragt. Zuhanden der IV-Behörde reichte der Arzt einen Bericht ein, wonach der Arbeitsunterbruch seiner Patientin nicht ausschliesslich aus gesundheitlichen Gründen erfolgt sei. Vielmehr habe die Frau ihre kleine Tochter stillen und betreuen wollen. Es gebe keine Gründe, die gegen die Fortführung ihres Berufes als Büroangestellte sprechen würden. Später bestätigte er gegenüber der IV, dass seine Patientin wieder fähig sei, zu 100\% zu arbeiten. Daraufhin wies die IV das Rentenbegehren der Patientin ab. In der Folge erhob die Taggeldversicherung Strafanzeige gegen den Arzt, nachdem sie vom Entscheid der IV Kenntnis erhalten und Einsicht in die Akten genommen hatte.

Im Strafverfahren räumte der Arzt ein, es sei medizinisch zwar gerechtfertigt gewesen, dass er seine Patientin (zuhanden der Taggeldversicherung) als arbeitsunfähig erklärt habe. Hingegen habe er mit der nachfolgenden (wahrheitswidrigen) Bescheinigung der Erwerbsfähigkeit gegenüber der IV ausschliesslich die Interessen seiner Patientin wahren wollen. Seiner Ansicht nach habe die Aussicht auf eine Rente jeweils negative Auswirkungen auf den Therapieerfolg der Patienten. Er sei immer davon ausgegangen, dass er niemanden schädigen würde. Das Bundesgericht führte dazu aus, für eine Bestrafung nach Art. 318 StGB genüge es, dass das Zeugnis «zum Gebrauche bei einer Behörde» bestimmt sei. Dies sei erfüllt, weil der Arzt den (unwahren) Bericht der IV-Behörde eingereicht habe. Es könne vorliegend auch nicht - wie vom Arzt geltend gemacht - von Fahrlässigkeit (und ebenfalls nicht von einem Irrtum über die Rechtswidrigkeit im Sinne von Art. $21 \mathrm{StGB}$ ) ausgegangen werden. Medizinische Gutachten seien ausschliesslich nach medizinischen Gesichtspunkten auszustellen. Sofern ein Arzt aus therapeutischen Gründen in ein Dilemma gerate, die Wahrheit über den Zustand eines Patienten zuhanden einer Behörde festzuhalten, müsse er die Begutachtung zurückweisen oder an einen Fachkollegen delegieren. Der Arzt wurde von der Vorinstanz zu einer bedingt vollziehbaren Strafe von 30 Tagessätzen à Fr. 500.- und einer Busse von Fr. 3000.- verurteilt [2]. Das Bundesgericht sprach den Arzt zwar im Grundsatz schuldig, wies die Sache bezüglich des Strafmasses jedoch an die Vorinstanz zur Neubeurteilung zurück.

\section{Bemerkungen}

Die beiden Entscheide sind in verschiedener Hinsicht bemerkenswert. Von höchstrichterlicher Instanz erfolgen erstmals zwei Verurteilungen aufgrund von Art. 318 StGB. Mit dieser Strafbestimmung soll das Vertrauen geschützt werden, das dem ärztlichen Zeugnis im allgemeinen Rechtsverkehr entgegengebracht 
wird. Daraus ergibt sich im wesentlichen zweierlei: Vom Arzt gefordert werden zum einen «wahre» Zeugnisse; mithin Zeugnisse [3], die ein zutreffendes Bild vom Gesundheitszustand des Menschen vermitteln. Dies gilt selbst dann, wenn der Arzt nachvollziehbare Beweggründe hat [4], im Interesse der Patientin ein «unwahres» Bild zu zeichnen. Zum andern ist nach Art. 318 StGB lediglich dasjenige Zeugnis relevant, das für einen besonderen Zweck bestimmt ist. Dabei wird aber nicht nur derjenige Arzt bestraft, der mit dem Zeugnis für sich selber einen Vorteil erzielen will. Ebenfalls wird geahndet, wenn mit dem Zeugnis unberechtigte Vorteile [5] zugunsten anderer Personen, typischerweise für den Patienten, erlangt oder Interessen Dritter verletzt werden können. Schliesslich genügt bereits, dass das Zeugnis zum Gebrauch bei einer Behörde bestimmt ist [6].

Die beiden Bundesgerichtsentscheide verdeutlichen die grosse Verantwortung, derer sich Ärzte beim Verfassen ihrer medizinischen Beurteilungen bewusst sein müssen. Dies gilt vor allem deshalb, weil der Inhalt eines Gutachtens sowie eines Zeugnisses und Berichtes weitreichende Konsequenzen haben kann und sich spätere Akteure auf die medizinischen Angaben sollen verlassen können. Insbesondere auch in Fällen, in welchen eigene Patienten (zu welchen Ärzte in einem Vertrauens- und Vertragsverhältnis stehen) beurteilt werden müssen, haben sich Ärzte eine uneingeschränkte Wahrheitstreue aufzuerlegen.

\section{Literatur}

1 Art. 34 Standesordnung der FMH.

2 Das Bundesgericht sprach den Arzt im Grundsatz schuldig, wies die Sache bezüglich des Strafmasses jedoch an die Vorinstanz zur Neubeurteilung zurück.

3 Schriftliche, mit Datum und Unterschrift versehene Erklärung einer Medizinalperson, dass ein gewisser Krankheitszustand bestehe oder eine gewisse medizinische Behandlungsweise aufgrund der vorgängigen Untersuchung angebracht sei oder dass aufgrund eines diagnostizierten körperlichen oder psychischen Zustandes eine Arbeitsunfähigkeit bestehe bzw. eine Beurlaubung angezeigt sei (Markus Boog, in: Basler Kommentar, Strafgesetzbuch II, 2. Aufl. Basel 2007, Art. 318 N 3 mit Hinweisen).

4 vgl. das oben besprochene Urteil 6B_152/2007 vom 13. Mai 2008.

5 z. B. zugunsten der Patientin; vgl. das oben besprochene Urteil 6B_99/2008 vom 18. März 2008.

6 Diese Zweckbestimmung kann sich aus dem Zeugnis selbst (Formular) oder aus den Absichten der Beteiligten im Einzelfall ergeben (Markus Boog, a.a. O., N 8 mit Hinweisen). 